\title{
Technology-Based Interventions for Nursing Home Residents: Implications for Nursing Home Practice Amid and Beyond the Influence of COVID-19: A Systematic Review Protocol
}

\section{Zhaohui Su ( $\nabla$ szh@utexas.edu )}

University of Texas Health Science Center at San Antonio https://orcid.org/0000-0003-2005-9504

Kylie Meyer

University of Texas Health Science Center at San Antonio

Yue Li

University of Rochester Medical Center

Dean McDonnell

Institute of Technology Carlow

Nitha Mathew Joseph

University of Texas Health Science Center at Houston

\section{Xiaoshan Li}

Beijing Normal University-Hong Kong Baptist University United International College

Yan Du

University of Texas Health Science Center at San Antonio

Shailesh Advani

National Institute of Health

Ali Cheshmehzangi

University of Nottingham

Junaid Ahmad

Peshawar Medical College

Claudimar Pereira da Veiga

Universidade Federal do Parana

Roger Yat-Nork Chung

University of Hong Kong

Jing Wang

University of Texas Health Science Center at San Antonio

Research 
Keywords: COVID-19, novel coronavirus, SARS-CoV2, nursing home residents, older adults, underserved populations, technology-based interventions, socio-ecological model

Posted Date: December 14th, 2020

DOI: https://doi.org/10.21203/rs.3.rs-56102/v2

License: (c) (1) This work is licensed under a Creative Commons Attribution 4.0 International License. Read Full License 


\section{Abstract}

Background: A growing number of technology-based interventions are used to support the health and quality of life of nursing home residents. The onset of COVID-19 and recommended social distancing policies that followed led to an increased interest in technology-based solutions to provide healthcare and promote health. Yet, there are no comprehensive resources on technology-based healthcare solutions that describe their efficacy for nursing home residents. This systematic review will identify technology-based interventions designed for nursing home residents and describe the characteristics and effects of these interventions concerning the distinctive traits of nursing home residents and nursing facilities.

Additionally, this paper will present practical insights into the varying intervention approaches that can assist in the delivery of broad digital health solutions for nursing home residents amid and beyond the impact of COVID-19.

Methods: Databases including PubMed, PsycINFO, CINAHL, and Scopus will be used to identify articles related to technology-based interventions for nursing home residents published between January $1^{\text {st }}$, 2010 to December $4^{\text {th }}, 2020$. Titles, abstracts, and full-texts papers will be reviewed against the eligibility criteria. The Preferred Reporting Items for Systematic Reviews and Meta-Analyses procedures will be followed for the reporting process, and implications for existing interventions and research evaluated by a multidisciplinary research team.

Results: NA-protocol study

Conclusions: Our study will fill critical gaps in the literature by providing a review of technology-based interventions tested in the nursing home setting. As the older adult population grows, there is an urgent need to identify effective technology-based interventions that can address the distinctive characteristics and preferences of nursing home residents. Clear and comprehensive understanding of how available technology-based health solutions facilitate healthcare for nursing home residents will shed light on the approaches open to residents to fend off the negative health consequences amid and beyond the influence of COVID-19.

\section{Systematic Review Registrations: PROSPERO CRD 42020191880}

\section{Background}

Nursing homes have been described as a "ground zero" throughout the coronavirus outbreak [1-4]. While the final impact of COVID-19 (coronavirus) on short and longer-term health outcomes is still unclear as the pandemic continues to unfold [5], what is clear is that nursing home residents have suffered some of the gravest consequences of this pandemic so far [6]. Contributing to over $40 \%$ of COVID- 19 deaths within the United States, 100,033 residents and workers in nursing homes have passed away from COVID19 (as of November $24^{\text {th }}, 2020$ ) [7]. Worse still is the fact that the nursing home residents who have died from COVID-19 mostly died without the care or company of their family members [8-10]. These staggering numbers underscore the urgent need for healthcare researchers to understand factors that 
make nursing home residents more vulnerable to COVID-19, and to identify practical solutions that can address these factors in a timely fashion.

Nursing homes and nursing facilities provide long-term service and support for individuals living with chronic or disabling conditions who are unable to live at home independently [11-13]. Often living with multiple morbidities, in the United States approximately $85.1 \%$ of nursing home residents are 65 years and older, with $75.8 \%$ of these individuals experiencing hypertension, $58.9 \%$ living with Alzheimer's Disease, and $53.0 \%$ of residents living with depression [13]. Three sets of factors likely contribute to the alarming COVID-19 death rates seen in nursing homes: (1) characteristics of nursing home residents [1418], (2) distinctive attributes of nursing home facilities [1, 6, 9, 10, 19], and (3) the micro and macro-level supports available to nursing home residents [2, 20-24]. On a micro level, research indicates that nursing home residents are more susceptible to infection and fatal outcomes from COVID-19 because they are often older adults living with medical conditions that compromise the immune systems and lowers their ability to combat the virus $[13,19,25,26]$. Additionally, they often lack specific self-care skills, such as utilizing telemedicine tools, or they may have a physical or cognitive impairment that impedes their ability to take care of their health and wellbeing [27-31]. The macro perspective focuses on the unique characteristics of nursing home facilities, as they are typically operating on a close and shared-living environment - conditions that are ideal for the spread of the virus [10, 19, 32]. Further, nursing homes often lack adequate healthcare resources or infrastructure needed to curb the impact of COVID-19. For example, numerous studies have indicated a lack of investment in training programs for nursing home staff, in addition to high turnover rates [33,34], that management teams are often ineffective [35, 36], and that the nursing home infrastructure is often too outdated [32, 37-39].

The third set of factors that contribute to nursing home residents' vulnerability to COVID-19 centers on social support available to these adults. Nursing home residents often have limited access to micro-level social support, including support from formal (e.g., doctors and nurses) or informal caregivers (e.g., family, friends, and acquaintances) [40], local community [41], and organizations (e.g., inexperienced or inadequately trained staff) [42]. Furthermore, nursing home residents often have limited macro-level social support. This is evidenced by harmful social norms (e.g., age-related discrimination)[43-45] and inadequate policy support that facilitates healthy aging and quality of life (e.g., insufficient regulatory oversight to ensure quality care in nursing homes) $[4,20,46,47]$. These factors combined, could result in severe health consequences in nursing home residents, such as wide viral spread [1].

The above areas of inquiry resonate with the core principles of the bioecological model [48-51], which highlights the way individuals are influenced by a series of synergistic interactions between intrapersonal and interpersonal factors (e.g., residents, resident families), organizational characteristics (e.g., nursing homes), policy (e.g., legislative response) and the social/community (e.g., ageism) context, and how these processes can change over time (See Figure 1.) To successfully and effectively protect nursing home residents from global health crises like COVID-19, stakeholders such as policymakers, healthcare professionals, informal caregivers, and older adults themselves all need to contribute to the changemaking process $[2,46,52,53]$. While some effective changes are resource-intensive, time-consuming, and 
need concerted efforts from multilevel stakeholders to achieve, there are cost-effective, efficient, and accessible health solutions available to nursing home residents, such as technology-based interventions $[54,55]$.

Technology-based interventions can be considered as the use of technology (e.g., digital devices like tablets and wearable devices, communication platforms) to manage or support health promotion strategies that aim to produce accessible and affordable health solutions to a target audience. Compared to traditional health solutions, such as face-to-face consultations, technology-based interventions have the potential to deliver healthcare more effectively and can mitigate geographic and access-related limitations that, as studies show, can play a significant role within nursing homes [56-62]. The evidence further suggests that technology-based interventions can help free healthcare professionals from repetitive work and allow them to make more meaningful contributions in delivering healthcare solutions to the care recipients [63-65].

Telemedicine and other technology-based solutions are particularly crucial given circumstances rendered by the COVID-19 pandemic, such as the limited ability for some healthcare providers to enter residences or for residents to visit their healthcare team for primary care visits. Limiting the exposure to infection through the use of telemedicine may assist in situations where a resident is required to attend a hospital appointment and return to a residence, thus alleviating the potential risk for a virus to spread to others [66]. Further, technologies that support residents' ability to remain in contact with families and friends outside of skilled care settings may reduce the adverse effects of loneliness and social isolation that are more common among nursing home residents compared to community-dwelling older adults $[17,67]$.

While technology-based solutions have potential to deliver health solutions to nursing home residents [68, 69], there is limited awareness of the benefits and delivery options for state-of-art technology-based interventions specifically designed for nursing home residents. By factoring for the distinctive characteristics of nursing home residents and nursing home facilities, the main focus of this systematic review is to identify and evaluate technology-based interventions tailored explicitly for nursing home residents. Additionally, this research will present practical insights into the varying intervention approaches that can assist in the delivery of broad digital health solutions for nursing home residents amid and beyond the impact of COVID-19. Overall, our research aims are:

- To identify technology-based interventions designed for nursing home residents and describe the characteristics and effects of these interventions concerning the distinctive traits of nursing home residents and nursing facilities.

- To present practical insights into the varying intervention approaches that can assist in the delivery of broad digital health solutions for nursing home residents amid and beyond the impact of COVID19.

\section{Methods}


The Preferred Reporting Items for Systematic Reviews and Meta-Analyses (PRISMA) procedures will be adhered to in the reporting process [70]. This systematic review is registered with the International Prospective Register of Systematic Reviews (PROSPERO) system (CRD 42020191880); these measures are to avoid unnecessary study duplication [71, 72], increase research rigor [73, 74], improve study comparability and replicability [75], and ultimately, promote quality and transparency in research [76]. The review being carried out in line with the Cochrane Handbook [77].

\section{Inclusion and exclusion criteria}

Based on the research aims, inclusion criteria were set a priori (Table 1). Articles published between January $1^{\text {st }}, 2010$ to December $4^{\text {th }}, 2020$ were included in the review. This period of time was selected to ensure a focus on up-to-date technologies, given the tendency of technology-based interventions to evolve and become out-of-date. The timeline will also make sure both studies conducted before and amid COVID-19 will be included in the review. In this study, nursing home residents are defined as people "having a length of stay in a nursing home for more than 90 days at any point" [78]. Technology-based interventions are defined as "the use of technology to manage or support health promotion strategies that aim to produce accessible and affordable health solutions to a target audience" [79]. Articles will be excluded if (1) the study sample did not include a majority nursing home residents (i.e., nursing home residents $\leq 50 \%$ of the total research population), (2) the study did not include and discuss technologybased health solutions designed for nursing home residents, (3) the study did not adopt a randomized controlled trial design (e.g., studies with quasi-experimental design were excluded), and (4) the authors did not report original empirical findings on intervention outcomes (e.g., research protocols and systematic review studies were excluded from the review).

Table 1. Study inclusion criteria 


\begin{tabular}{|c|c|}
\hline Category & Criteria \\
\hline Study population & Adults ( $\geq 18$ years old) living in nursing homes \\
\hline Language & English and Chinese \\
\hline $\begin{array}{l}\text { Intervention/health } \\
\text { solution }\end{array}$ & $\begin{array}{l}\text { Technology-based interventions (e.g., digital tools such as smartphones } \\
\text { and tablets, sensor devices, internet-based programs) }\end{array}$ \\
\hline Key variable & $\begin{array}{l}\text { Detailed descriptions of the technology-based interventions (i.e., } \\
\text { purpose of the intervention (to evaluate the aims of existing } \\
\text { interventions), use of technology (to examine how different types of } \\
\text { technologies have been applied among nursing home residents), } \\
\text { application of the interventions (to investigate the degree to which the } \\
\text { interventions have involved nursing home residents in the } \\
\text { adoption/application of technologies), intervention exposure (to study } \\
\text { how different interventions have been used among nursing home } \\
\text { residents), outcome variables assessed/measured (to evaluate the } \\
\text { quantifiable outcomes of technology-based interventions), and weather } \\
\text { the design of the intervention material is tailored to nursing home } \\
\text { residents (to examine to degree to which the design technology-based } \\
\text { interventions has taken the unique attributes of nursing home residents } \\
\text { into consideration) }\end{array}$ \\
\hline Study type & $\begin{array}{l}\text { Original research (i.e., research that reports original and empirical } \\
\text { research findings) }\end{array}$ \\
\hline Study design & Randomized controlled trials \\
\hline Study outcome & $\begin{array}{l}\text { Empirical reporting of the effect of the intervention (i.e., qualitative } \\
\text { designs excluded) }\end{array}$ \\
\hline
\end{tabular}

\section{Search strategy}

Databases including PubMed, PsycINFO, CINAHL, and Scopus, will be searched for eligible articles. We will also search ProQuest Dissertations to examine gray literature sources. A search strategy was developed in consultation with a librarian experienced in systematic review methods. Search terms used to locate articles will center on three concepts: nursing home residents, technology-based interventions, and randomized controlled trials. An example PubMed search string is illustrated in Table 2.

Table 2. Example PubMed search string 


\section{Concept Search string}

\begin{tabular}{|c|c|}
\hline $\begin{array}{l}\mathrm{ng} \\
\mathrm{s}\end{array}$ & 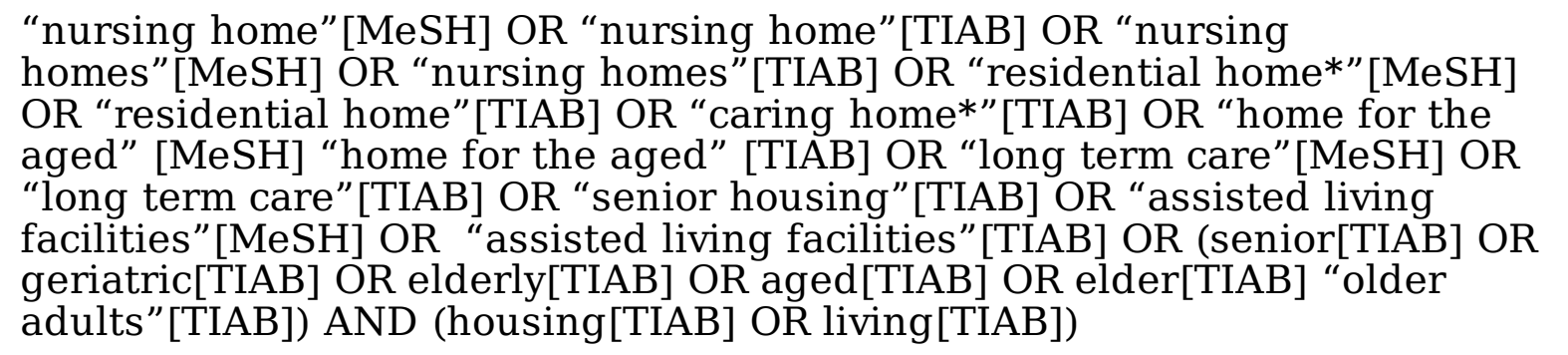 \\
\hline $\begin{array}{l}\text { chnology- } \\
\text { sed } \\
\text { terventions }\end{array}$ &  \\
\hline
\end{tabular}

randomized controlled trial[PT] OR randomized controlled trials as topic[MH]

Randomized controlled trials

OR random allocation [MH] OR double-blind method[MH] OR single-blind method[MH] OR random*[tw] OR "Placebos"[MeSH] OR placebo[TIAB] OR ((singl*[tw] OR doubl*[tw] OR trebl*[TW] OR tripl*[TW]) AND (mask*[TW] OR blind*[TW] OR dumm*[TW]))

\section{Study selection}

Following the search, all citations will be collated and uploaded to Mendeley, and duplicate studies will be removed. Titles and then abstracts will be screened by two principal reviewers independently. The same screening procedure will be adopted in the full-text article review process on selected article abstracts. Reasons for exclusion will be recorded and detailed in the PRISMA flowchart. Discrepancies between reviewers will be resolved via group discussions using videoconferencing and email correspondence to reach a consensus.

\section{Study quality assessment}

The Cochrane Collaboration evaluation framework will be adopted to examine risk of bias of the included study [80]. The framework has seven domains: random sequence generation, allocation concealment, blinding of participants and personnel, blinding of outcome assessment, incomplete outcome data, selective reporting, and any other source of bias. The risk of bias will be evaluated independently by two reviewers (ZS and XL), who will qualitatively evaluate risk of bias and provide a score (high, medium, low). Any discrepancy regarding the risk of bias will be resolved by consensus via group discussions. Both junior and senior authors will be involved in the discrepancy review process, wherein, if agreement cannot be reached, the senior author will decide on the appropriate rating.

\section{Data extraction and synthesis}


Data on study design, sample characteristics (i.e., sample size and sample details), intervention characteristics (i.e., technology type, intervention application, intervention exposure, and intervention materials), outcome variables assessed, and research findings will be extracted by two main reviewers (ZS and $X L$ ). Findings from the included studies will be narratively synthesized to examine the characteristics and effects of the interventions. Gaining a more structured understanding of the interventions, the multidisciplinary study team will organize insights on intervention application and outcomes in tandem with the distinctive traits of nursing home residents and the overall nursing home environment. Due to the heterogeneity found within the articles identified during a preliminary review of search results, meta-analyses are not considered.

\section{Results}

NA: As this is a protocol study

\section{Discussion}

There is a growing body of technology-based interventions designed to support the health and quality of life of nursing home residents [56-62]. The onset of COVID-19 and recommended social distancing policy led to an increased interest in reliance on technology-based solutions [81, 82]. However, research has yet to provide comparative insight into the recent state of development of these interventions and how current evidence apply in the context of the COVID-19 pandemic. The use of the socio-ecological model, combined with multidisciplinary expertise, provides a framework to present practical insights on how these interventions can be utilized to deliver health solutions to nursing home residents amid and beyond the impact of COVID-19.

This research fills a critical gap in the literature by consolidating, in one place, the evidence for technology-based interventions empirically tested with nursing home populations. As the older adult population grows, there is an urgent need to identify effective technology-based interventions that can address the distinctive characteristics and preferences of nursing home residents [83, 84]. Improving person-centered care and the delivery of effective care solutions to nursing home residents, especially as the pandemic continues, is of critical importance. Comprehensive understanding of how available technology-based health solutions facilitate healthcare for nursing home residents can help shed light on approaches that are available to these residents to fend off the negative health consequences amid and beyond the influence of COVID-19. While the COVID-19 pandemic has revealed troubling vulnerabilities in the long-term care system across the globe, it also shows how telemedicine can support nursing home residents and their families. Technology can also assist clinicians in connecting with patients when inperson medical visits are difficult or dangerous (e.g., in rural settings, following natural disasters). Telemedicine and other technology-based interventions have the potential to provide a comprehensive range of benefits. This research also serves as a platform for care institutions and policymakers to inform government policies and further justify the role technology can play in strengthening the service provision across nursing homes and facilities. 


\section{Conclusions}

NA: As this is a protocol study

\section{List Of Abbreviations}

COVID-19: covronavirus disease 2019

\section{Declarations}

Ethics approval and consent to participate

Not applicable.

Consent for publication

Not applicable.

Availability of data and materials

No

Competing interests

None

Funding

None

Authors' contributions

ZS developed the research idea and wrote and manuscript. KM, YL, DMD, NMJ, XL, YD, SA, AC, JA, CPV, YNC, and JW reviewed and revised the manuscript.

\section{Acknowledgements}

The authors wish to express their gratitude to Emme Lopez, the academic librarian who helped with finalizing the search strategy. Gratitude also goes to the editors and reviewers for their constructive input.

\section{References}

1. Barnett, M.L. and D.C. Grabowski, Nursing homes are ground zero for COVID-19 pandemic. JAMA Health Forum, 2020. 1(3): p. e200369-e200369. 
2. Pillemer, K., L. Subramanian, and N. Hupert, The importance of long-term care populations in models of COVID-19. JAMA, 2020.

3. Benson, J.J., et al., Online social support groups for informal caregivers of hospice patients with cancer. European Journal of Oncology Nursing, 2020. 44: p. 101698.

4. Li, Y., et al., COVID-19 infections and deaths among Connecticut nursing home residents: Facility correlates. Journal of the American Geriatrics Society, 2020. n/a(n/a).

5. Cutler, D., How will COVID-19 affect the health care economy? JAMA Health Forum, 2020. 1(4): p. e200419-e200419.

6. Fallon, A., et al., COVID-19 in nursing homes. QJM, 2020: p. hcaa136.

7. Kaiser Family Foundation, State data and policy actions to address coronavirus. 2020, Kaiser Family Foundation.

8. Davidson, P.M. and S.L. Szanton, Nursing homes and COVID-19: We can and should do better. Journal of Clinical Nursing, 2020. n/a(n/a).

9. Comas-Herrera, A., et al., Mortality associated with COVID-19 outbreaks in care homes: Early international evidence. International Long Term Care Policy Network, 2020.

10. McMichael, T.M., et al., Epidemiology of Covid-19 in a long-term care facility in King County, Washington. New England Journal of Medicine, 2020. 382(21): p. 2005-2011.

11. Backman, A., et al., Characteristics of highly rated leadership in nursing homes using item response theory. Journal of Advanced Nursing, 2017. 73(12): p. 2903-2913.

12. National Institute for Aging. Residential facilities, assisted living, and nursing homes. 2017 [cited 2020 June 14]; Available from: https://www.nia.nih.gov/health/residential-facilities-assisted-livingand-nursing-homes.

13. U.S. Department of Health And Human Services, Long-term care providers and services users in the United States, 2015-2016, in Vital and Health Statistics. 2019, Centers for Disease Control and Prevention,..

14. Dowd, J.B., et al., Demographic science aids in understanding the spread and fatality rates of COVID19. Proceedings of the National Academy of Sciences, 2020. 117(18): p. 9696.

15. CDC COVID-19 Response Team, Severe outcomes among patients with coronavirus disease 2019 (COVID-19)-United States, February 12-March 16, 2020. MMWR Morb Mortal Wkly Rep, 2020. 69(12): p. 343-346.

16. Wherton, J., et al., Designing assisted living technologies 'in the wild': Preliminary experiences with cultural probe methodology. BMC Med Res Methodol, 2012. 12: p. 188.

17. Hado, E. and L. Friss Feinberg, Amid the COVID-19 pandemic, meaningful communication between family caregivers and residents of long-term care facilities is imperative. Journal of Aging \& Social Policy, 2020: p. 1-6.

18. Liu, Y.-B., et al., Social support, health literacy, and health care utilization among older adults. Asia Pacific Journal of Public Health, 2019. 31(4): p. 359-366. 
19. Etard, J.-F., et al., Potential lethal outbreak of coronavirus disease (COVID-19) among the elderly in retirement homes and long-term facilities, France, March 2020. Euro surveillance : bulletin Europeen sur les maladies transmissibles = European communicable disease bulletin, 2020. 25(15): $p$. 2000448.

20. Gurwitz, J.H., COVID-19, post-acute care preparedness and nursing homes: Flawed policy in the fog of war. Journal of the American Geriatrics Society, 2020: p. 10.1111/jgs.16499.

21. Laditka, S.B., et al., Providing shelter to nursing home evacuees in disasters: Lessons from Hurricane Katrina. American Journal of Public Health, 2008. 98(7): p. 1288-1293.

22. Schott, N. and M. Tietjens, Exploring the mediating role of social support and fall efficacy on the association between falls and physical activity: A cross-sectional study in an assisted-living population. J Aging Phys Act, 2019. 27(1): p. 53-60.

23. Grabowski, D.C. and K.E. Joynt Maddox, Postacute care preparedness for COVID-19: Thinking ahead. JAMA, 2020. 323(20): p. 2007-2008.

24. White, E.M., L.H. Aiken, and M.D. McHugh, Registered nurse burnout, job dissatisfaction, and missed care in nursing homes. Journal of the American Geriatrics Society, 2019. 67(10): p. 2065-2071.

25. Applegate, W.B. and J.G. Ouslander, COVID-19 presents high risk to older persons. Journal of the American Geriatrics Society, 2020. 68(4): p. 681-681.

26. Centers for Disease Control and Prevention. Coronavirus disease 2019 (COVID-19): Older adults. 2020; Available from: https://www.cdc.gov/coronavirus/2019-ncov/need-extra-precautions/olderadults.html.

27. Scott Kruse, C., et al., Evaluating barriers to adopting telemedicine worldwide: A systematic review. Journal of Telemedicine and Telecare, 2016. 24(1): p. 4-12.

28. Nouri, S., et al., Addressing equity in telemedicine for chronic disease management during the Covid19 pandemic. NEJM Catalyst Innovations in Care Delivery, 2020. 1(3).

29. Berkowsky, R.W. and S.J. Czaja, Challenges associated with online health information seeking among older adults, in Aging, Technology and Health, R. Pak and A.C. McLaughlin, Editors. 2018, Academic Press: San Diego. p. 31-48.

30. Piper, A.M., R. Brewer, and R. Cornejo, Technology learning and use among older adults with late-life vision impairments. Universal Access in the Information Society, 2017. 16(3): p. 699-711.

31. Cutilli, C.C., et al., Health literacy, health disparities, and sources of health information in U.S. older adults. Orthopaedic Nursing, 2018. 37(1).

32. Benson, N.M., D. Öngür, and J. Hsu, COVID-19 testing and patients in mental health facilities. The Lancet. Psychiatry, 2020. 7(6): p. 476-477.

33. Fosse, A., et al., Nursing home physicians' assessments of barriers and strategies for end-of-life care in Norway and the Netherlands. Journal of the American Medical Directors Association, 2017. 18(8): p. 713-718. 
34. Quigley, D.D., et al., COVID-19 preparedness in nursing homes in the midst of the pandemic. Journal of the American Geriatrics Society, 2020: p. 10.1111/jgs.16520.

35. Glette, M.K., et al., Nursing home leaders' and nurses' experiences of resources, staffing and competence levels and the relation to hospital readmissions - a case study. BMC Health Serv Res, 2018. 18(1): p. 955.

36. Vaughn, V.M., et al., Characteristics of healthcare organisations struggling to improve quality: Results from a systematic review of qualitative studies. BMJ Quality \& Safety, 2019. 28(1): p. 74.

37. Solis, J., et al., Structural vulnerability in the United States revealed in three waves of novel coronavirus disease (COVID-19). The American Journal of Tropical Medicine and Hygiene, 2020: $p$. tpmd200391.

38. Zimmerman, S., et al., The need to include assisted living in responding to the COVID-19 pandemic. Journal of the American Medical Directors Association, 2020. 21(5): p. 572-575.

39. Khan, F. and J. Driessen, Bridging the telemedicine infrastructure gap: Implications for long-term care in rural America. Public Policy \& Aging Report, 2018. 28(3): p. 80-84.

40. Miller, V.J., Investigating barriers to family visitation of nursing home residents: A systematic review. Journal of Gerontological Social Work, 2019. 62(3): p. 261-278.

41. Stone, P.W., et al., The expansion of National Healthcare Safety Network enrollment and reporting in nursing homes: Lessons learned from a national qualitative study. American Journal of Infection Control, 2019. 47(6): p. 615-622.

42. Hales, C., et al., The care of older adults with extreme obesity in nursing homes: A collective case study. medRxiv, 2019: p. 19013326.

43. Jimenez-Sotomayor, M.R., C. Gomez-Moreno, and E. Soto-Perez-de-Celis, Coronavirus, ageism, and Twitter: An evaluation of tweets about older adults and COVID-19. Journal of the American Geriatrics Society, 2020. $\mathrm{n} / \mathrm{a}(\mathrm{n} / \mathrm{a})$.

44. Han, S.D. and L. Mosqueda, Elder abuse in the COVID-19 era. Journal of the American Geriatrics Society, 2020: p. 10.1111/jgs.16496.

45. Fraser, S., et al., Ageism and COVID-19: What does our society's response say about us? Age and ageing, 2020: p. afaa097.

46. Comas-Herrera, A., et al., COVID-19: Implications for the support of people with social care needs in England. Journal of Aging \& Social Policy, 2020: p. 1-8.

47. Ko, M., L. Wagner, and J. Spetz, Nursing home implementation of health information technology: Review of the literature finds inadequate investment in preparation, infrastructure, and training. INQUIRY: The Journal of Health Care Organization, Provision, and Financing, 2018. 55: p. 0046958018778902.

48. Bronfenbrenner, U., Toward an experimental ecology of human development. American Psychologist, 1977. 32(7): p. 513-531. 
49. Bronfenbrenner, U., The ecology of human development: Experiments by nature and by design. 1979, Cambridge, MA: Harvard University Press.

50. McLeroy, K.R., et al., An ecological perspective on health promotion programs. Health Education Quarterly, 1988. 15(4): p. 351-377.

51. Bronfenbrenner, U. and P. Morris, A., The bioecological model of human development, in Volume 1: Theoretical Models of Human Development. 2007: Handbook of child psychology.

52. Cooper, E., et al., Priorities for the professional development of registered nurses in nursing homes: $A$ Delphi study. Age and Ageing, 2016. 46(1): p. 39-45.

53. Cutter, C.M., C. Nelson, and M. Abir, Accountability to population health in the COVID-19 pandemic: Designing health care delivery within a social responsibility framework. Population Health Management, 2020.

54. Chiu, C.-J. and C.H. Wu, Information and communications technology as a health promotion method for older adults in assisted-living facilities: Three-arm group-randomized trial. JMIR Aging, 2019. 2(1): p. e12633.

55. Banbury, A., et al., Adding value to remote monitoring: Co-design of a health literacy intervention for older people with chronic disease delivered by telehealth - The telehealth literacy project. Patient Education and Counseling, 2020. 103(3): p. 597-606.

56. Ohannessian, R., T.A. Duong, and A. Odone, Global telemedicine implementation and integration within health systems to fight the COVID-19 pandemic: A call to action. JMIR Public Health Surveill, 2020. 6(2): p. e18810.

57. Smith, A.C., et al., Telehealth for global emergencies: Implications for coronavirus disease 2019 (COVID-19). Journal of Telemedicine and Telecare, 2020: p. 1357633X20916567.

58. Wang, J., et al., Connecting smartphone and wearable fitness tracker data with a nationally used electronic health record system for diabetes education to facilitate behavioral goal monitoring in diabetes care: Protocol for a pragmatic multi-site randomized trial. JMIR Res Protoc, 2018. 7(4): p. e10009.

59. Zhou, X., et al., The role of telehealth in reducing the mental health burden from COVID-19. Telemedicine and e-Health, 2020. 26(4): p. 377-379.

60. Mette, L.A., et al., Reaching high-risk underserved individuals for cancer genetic counseling by videoteleconferencing. J Community Support Oncol, 2016. 14(4): p. 162-8.

61. Hollander, J.E. and B.G. Carr, Virtually perfect? Telemedicine for Covid-19. New England Journal of Medicine, 2020. 382(18): p. 1679-1681.

62. Wang, J., et al., A behavioral lifestyle intervention enhanced with multiple-behavior self-monitoring using mobile and connected tools for underserved individuals with type 2 diabetes and comorbid overweight or obesity: Pilot comparative effectiveness trial. JMIR mHealth uHealth, 2018. 6(4): p. e92.

63. Keyworth, C., et al., What maximizes the effectiveness and implementation of technology-based interventions to support healthcare professional practice? A systematic literature review. BMC 
Medical Informatics and Decision Making, 2018. 18(1): p. 93.

64. Ehn, M., A.-C. Johansson, and Å. Revenäs, Technology-based motivation support for seniors' physical activity-A qualitative study on seniors' and health care professionals' views. International Journal of Environmental Research and Public Health, 2019. 16(13).

65. Bagot, K.L., et al., Sustainable implementation of innovative, technology-based health care practices: A qualitative case study from stroke telemedicine. Journal of Telemedicine and Telecare, 2018. 26(12): p. 79-91.

66. Naylor, M.D., K.B. Hirschman, and K. McCauley, Meeting the transitional care needs of older adults with COVID-19. Journal of Aging \& Social Policy, 2020. 32(4-5): p. 387-395.

67. Prieto-Flores, M.E., et al., Factors associated with loneliness of noninstitutionalized and institutionalized older adults. J Aging Health, 2011. 23(1): p. 177-94.

68. Mahmood, S., et al., Global preparedness against COVID-19: We must leverage the power of digital health. JMIR Public Health Surveill, 2020. 6(2): p. e18980.

69. Banskota, S., M. Healy, and E.M. Goldberg, 15 smartphone apps for older adults to use while in isolation during the COVID-19 pandemic. West J Emerg Med, 2020. 21(3): p. 514-525.

70. Moher, D., et al., Preferred Reporting Items for Systematic Reviews and Meta-Analyses: The PRISMA statement. PLoS Medicine, 2009. 6(7): p. e1000097.

71. Stewart, L., D. Moher, and P. Shekelle, Why prospective registration of systematic reviews makes sense. Syst Rev, 2012. 1: p. 7.

72. Chang, S.M. and J. Slutsky, Debunking myths of protocol registration. Syst Rev, 2012. 1: p. 4.

73. Xu, C., et al., Protocol registration or development may benefit the design, conduct and reporting of dose-response meta-analysis: Empirical evidence from a literature survey. BMC Medical Research Methodology, 2019. 19(1): p. 78.

74. dos Santos, M.B.F., et al., Protocol registration improves reporting quality of systematic reviews in dentistry. BMC Medical Research Methodology, 2020. 20(1): p. 57.

75. Shokraneh, F., Reproducibility and replicability of systematic reviews. World Journal of MetaAnalysis, 2019. 7(3).

76. Ramstrand, N., et al., Promoting quality and transparency in clinical research. Prosthetics and Orthotics International, 2019. 43(5): p. 474-477.

77. Higgins, J.P.T., et al., The Cochrane Collaboration's tool for assessing risk of bias in randomised trials. BMJ, 2011. 343: p. d5928.

78. Thomas, K.S., et al., A methodology to identify a cohort of Medicare beneficiaries residing in large assisted living facilities using administrative data. Medical Care, 2018. 56(2): p. e10-e15.

79. Su, Z., et al., Understanding technology-based interventions for caregivers of cancer patients: $A$ systematic review-based concept analysis. Journal of Medical Internet Research, 2020.

80. Higgins, J.P., et al., Cochrane handbook for systematic reviews of interventions. 2019: John Wiley \& Sons. 
81. Goodman-Casanova, J.M., et al., Telehealth home support during COVID-19 confinement for community-dwelling older adults with mild cognitive impairment or mild dementia: Survey study. J Med Internet Res, 2020. 22(5): p. e19434.

82. Gao, Z., et al., Virtual reality exercise as a coping strategy for health and wellness promotion in older adults during the COVID-19 pandemic. Journal of Clinical Medicine, 2020. 9(6).

83. Schneider, J.E., et al., Economic evaluation of passive monitoring technology for seniors. Aging Clin Exp Res, 2020. 32(7): p. 1375-1382.

84. Sapci, A.H. and H.A. Sapci, Innovative assisted living tools, remote monitoring technologies, artificial intelligence-driven solutions, and robotic systems for aging societies: Systematic review. JMIR Aging, 2019. 2(2): p. e15429.

\section{Figures}

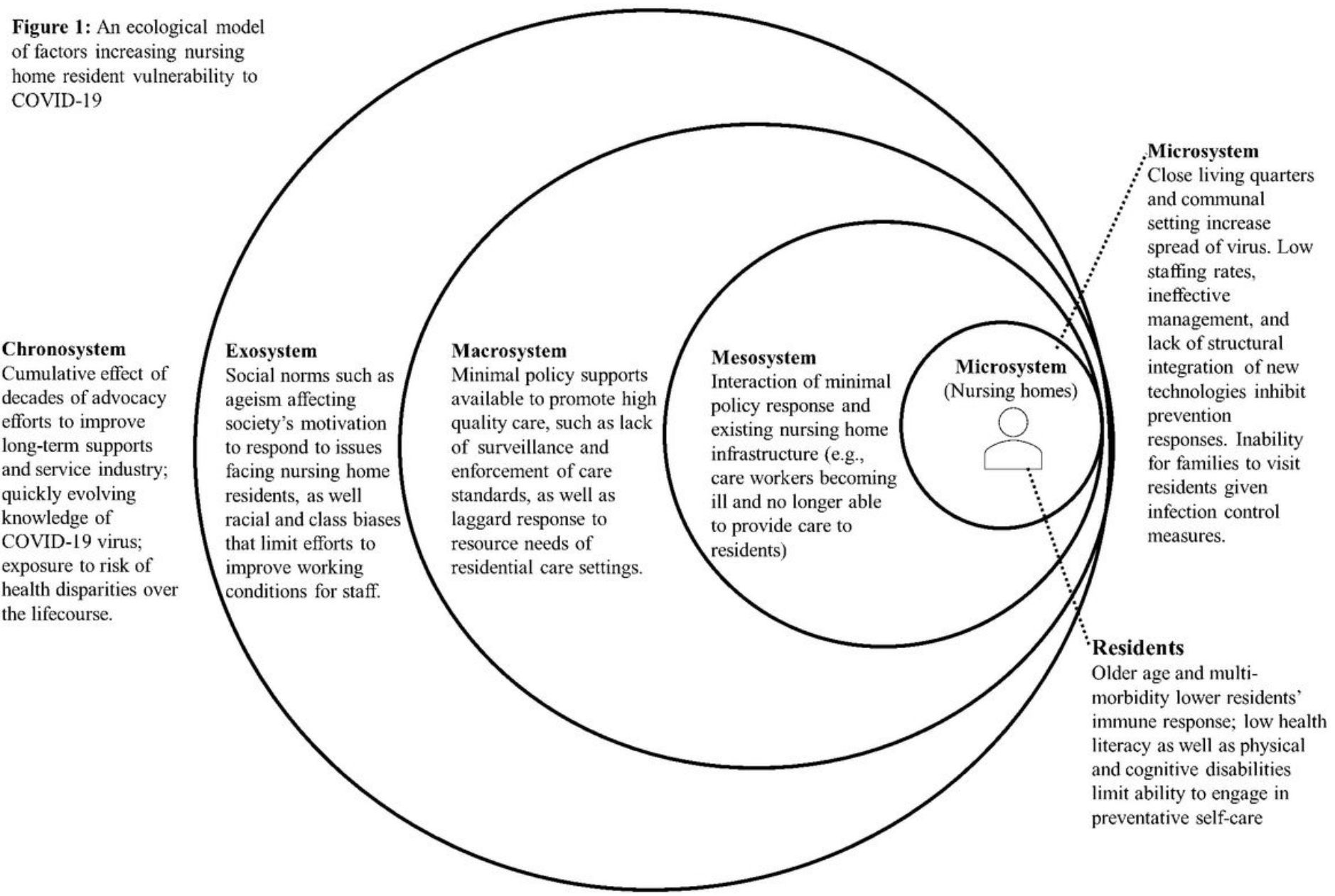

Figure 1

An ecological model of factors increasing nursing home resident vulnerability to COVID-19

\section{Supplementary Files}


This is a list of supplementary files associated with this preprint. Click to download.

- NursingHomePRISMA2009checklist.doc 